\title{
Clinically Relevant Imaging Features for MGMT Promoter Methylation in Multiple Glioblastoma Studies: A Systematic Review and Meta-Analysis
}

\author{
(D)C.H. Suh, DH.S. Kim, DS.C. Jung, (D)C.G. Choi, and DS.J. Kim

\begin{abstract}
BACKGROUND: O6-methylguanine methyltransferase (MGMT) promoter methylation status has been reported as a prognostic biomarker in clinical trials.

PURPOSE: Our aim was to systematically evaluate imaging features of MGMT promoter methylated glioblastoma and to determine the diagnostic performance of MR imaging for prediction of MGMT promoter methylation in patients with newly diagnosed glioblastoma.
\end{abstract}

DATA SOURCES: A computerized search of Ovid MEDLINE and EMBASE up to February 27, 2018, was conducted.

STUDY SELECTION: We selected studies evaluating imaging features of MGMT promoter methylated glioblastoma and the diagnostic performance of MR imaging for prediction of MGMT promoter methylation.

DATA ANALYSIS: Pooled estimates of sensitivity and specificity were calculated using a hierarchic logistic regression model. Metaregression and sensitivity analysis were performed.

DATA SYNTHESIS: Twenty-two articles including 2199 patients were included. MGMT promoter methylated glioblastoma is likely to show less edema, high ADC, and low perfusion. Ten articles including 753 patients were included in the meta-analysis. The summary sensitivity was $79 \%$ ( $95 \% \mathrm{Cl}, 72 \%-85 \%)$, and the summary specificity was $78 \%$ (95\% Cl, $71 \%-84 \%$ ). In the meta-regression, MGMT promoter methylation and mean age were associated with heterogeneity. Sensitivity analysis excluding 1 study resolved the heterogeneity.

LIMITATIONS: Included studies used a variety of different MR imaging techniques to predict MGMT promoter methylation.

CONCLUSIONS: MGMT promotor methylated glioblastoma is likely to show less aggressive imaging features than MGMT promotor unmethylated glioblastoma. Despite the variety of different MR imaging techniques used, MR imaging in patients with newly diagnosed glioblastoma was shown to have the potential to predict MGMT promoter methylation noninvasively.

ABBREVIATIONS: $D C E=$ dynamic contrast-enhanced imaging; $\mathrm{HSROC}=$ hierarchic summary receiver operating characteristic; $I D H=$ isocitrate dehydrogenase; MGMT = O6-methylguanine methyltransferase; PRISMA = Preferred Reporting Items for Systematic Reviews and Meta-Analyses; QUADAS-2 = Quality Assessment of Diagnostic Accuracy Studies-2; WHO = World Health Organization

$\mathbf{E}$ pigenetic silencing of the O6-methylguanine methyltransferase (MGMT) gene by promotor methylation compromises DNA repair, ${ }^{1}$ and $M G M T$ promoter methylation status has been identified as a predictive biomarker for an alkylating chemotherapy response. ${ }^{2,3}$ In addition, MGMT promoter methylation status has been reported as a prognostic biomarker in clinical trials, ${ }^{4,5}$ and a

Received November 30, 2017; accepted after revision May 8, 2018.

From the Department of Radiology and Research Institute of Radiology, University of Ulsan College of Medicine, Asan Medical Center, Seoul, Republic of Korea.

This work was supported by a grant from the National R\&D Program for Cancer Control, Ministry of Health and Welfare, Republic of Korea (1720030).

Please address correspondence to Ho Sung Kim, MD, PhD, Department of Radiology and Research Institute of Radiology, University of Ulsan College of Medicine, Asan Medical Center, 86 Asanbyeongwon-Gil, Songpa-Gu, Seoul 138-736, Republic of Korea; e-mail: radhskim@gmail.com molecular-based recursive partitioning analysis model has recently been introduced as a potentially superior stratification variable in a clinical trial. ${ }^{6}$ Moreover, there is increasing evidence of the value of temozolomide monotherapy as a reasonable alternative to radiation therapy in elderly patients with MGMT promoter methylation. ${ }^{7,8}$ Furthermore, MGMT promoter methylation status has been reported as a strong predictor of pseudoprogression. ${ }^{9}$

Methylation-specific polymerase chain reaction of histopathologic specimens is considered a criterion standard method

\footnotetext{
-- Indicates open access to non-subscribers at www.ajnr.org

三 Indicates article with supplemental on-line tables.

Indicates article with supplemental on-line photos.

http://dx.doi.org/10.3174/ajnr.A5711
}

AJNR Am J Neuroradiol 39:1439-45 Aug 2018 www.ajnr.org 
for the diagnosis of MGMT promoter methylation. ${ }^{2}$ However, tissue-based genomics or epigenomics is generally invasive, and spatial and/or temporal intratumoral heterogeneity may reduce the value of invasive tissue-based genomic or epigenomic analysis. In cases of glioblastoma, a repeat operation or tissue sampling is also rarely performed at tumor recurrence. ${ }^{10}$ These tumor heterogeneities may provide opportunities for medical imaging, which could characterize the entire tumor in a noninvasive and repeatable way. Therefore, noninvasive, comprehensive, and repetitive assessments to predict MGMT promoter methylation may have great potential in routine clinical practice and may help in assessing prognosis or treatment response in patients with newly diagnosed glioblastoma.

Several studies have reported the imaging features and/or the diagnostic performance of MR imaging for the prediction of MGMT promoter methylation in patients with newly diagnosed glioblastoma. ${ }^{11-32}$ A diverse array of MR imaging modalities, including conventional MR imaging, diffusion-weighted imaging, diffusion tensor imaging, dynamic susceptibility-weighted contrast-enhanced imaging, dynamic contrast-enhanced imaging (DCE), arterial spin-labeling, susceptibility-weighted imaging, and amide proton transfer, have been used for such purposes. Recently, radiomics approaches using high-throughput quantitative imaging features have also been reported from various institutions. ${ }^{13,14,17,18,31}$

However, the imaging prediction of MGMT promoter methylation has not yet been systematically evaluated, to our knowledge. Therefore, we systematically evaluated the imaging features of MGMT promoter methylated glioblastoma and determined the diagnostic performance of MR imaging for the prediction of MGMT promoter methylation in patients with newly diagnosed glioblastoma.

\section{MATERIALS AND METHODS}

This study was performed and reported in accordance with the Preferred Reporting Items for Systematic Reviews and MetaAnalyses (PRISMA) guidelines. ${ }^{33}$ We established the following research questions: What are the imaging features of MGMT methylated glioblastoma in patients with newly diagnosed glioblastoma, and what is the diagnostic performance of MR imaging for prediction of MGMT promoter methylation?

\section{Literature Search}

A computerized search of Ovid MEDLINE and EMBASE up to February 27, 2018, was conducted to identify studies evaluating the imaging features of MGMT promoter methylated glioblastoma and the diagnostic performance of MR imaging for the prediction of MGMT promoter methylation in patients with newly diagnosed glioblastoma. The search terms combined synonyms for "glioblastoma," "MGMT," and "MR imaging" as follows: ((glioblastoma $\left.{ }^{\star}\right)$ OR (glioma $\left.\left.{ }^{\star}\right)\right)$ AND ((methylguanine-DNAmethyltransferase) OR (O6-methylguanin-DNA-methyltransferase) OR (MGMT)) AND ((MR imaging) OR (MR imaging) OR (MR imaging)). The bibliographies of identified studies were screened manually to expand the search. The systematic search was limited to English-language publications.

\section{Inclusion Criteria}

Studies were included if they satisfied all of the following criteria: 1) patients with newly diagnosed glioblastoma (grade IV glioma based on the World Health Organization [WHO] classification criteria $\left.^{34,35}\right)$;2) patients who underwent preoperative MR imaging including conventional MR imaging, advanced MR imaging, or a radiomics approach using high-throughput quantitative imaging features; 3 ) a reference standard based on methylation-specific polymerase chain reaction for MGMT promoter methylation; and 4) sufficient detail for acquisition of the imaging features of MGMT promoter methylated glioblastoma or reconstruction of $2 \times 2$ tables for determination of the diagnostic performance of MR imaging for prediction of MGMT promoter methylation in patients with newly diagnosed glioblastoma.

\section{Exclusion Criteria}

Studies were excluded if they met any of following criteria: 1) case reports/series including $<10$ patients; 2 ) conference abstracts, reviews, editorials, letters, or comments; 3 ) a study population including patients with recurrent glioblastoma; 4) studies focusing on subjects in whom techniques other than the MR imaging were used to diagnose MGMT promoter methylation; and 5) studies with a partially overlapping population (for the meta-analysis). In the event of an overlapping population or study period, the largest and latest study population was used. Authors of the studies were contacted for provision of further data when $2 \times 2$ tables could not be calculated.

\section{Data Extraction and Quality Assessment}

A standardized form was used to extract the following information from the selected studies-1) patient characteristics: sample size, number of patients with MGMT promoter methylation, histology of underlying disease (WHO grade), mean age (range), and male/female ratio; 2) study characteristics: authors, publication year, institution, patient recruitment period, study design (prospective versus retrospective), study enrollment (consecutive versus nonconsecutive), the reference standard, interval between MR imaging and the reference standard, and blinding to the reference standard; 3) MR imaging characteristics: magnetic field strength, scanner manufacturer, scanner model, number of head coil channels, types of MR imaging techniques, specific technical parameters, imaging features of MGMT promoter methylated glioblastoma; 4) MR imaging interpretation: number of readers, reader experience, and blinding to the reference standard; and 5) cutoff values for diagnosing MGMT promoter methylation and diagnostic performance of MR imaging for prediction of MGMT promoter methylation in patients with newly diagnosed glioblastoma.

Methodologic quality assessment was evaluated according to the Quality Assessment of Diagnostic Accuracy Studies-2 (QUADAS-2) criteria. $^{36}$ The literature selection, data extraction, and quality assessment were conducted independently by 2 reviewers (C.H.S. and H.S.K.). If disagreement occurred, a third reviewer (S.J.K.) was consulted to reach a consensus.

\section{Data Synthesis and Analysis}

Identification of the imaging features of MGMT promoter methylated glioblastoma and the diagnostic performance of MR imag- 


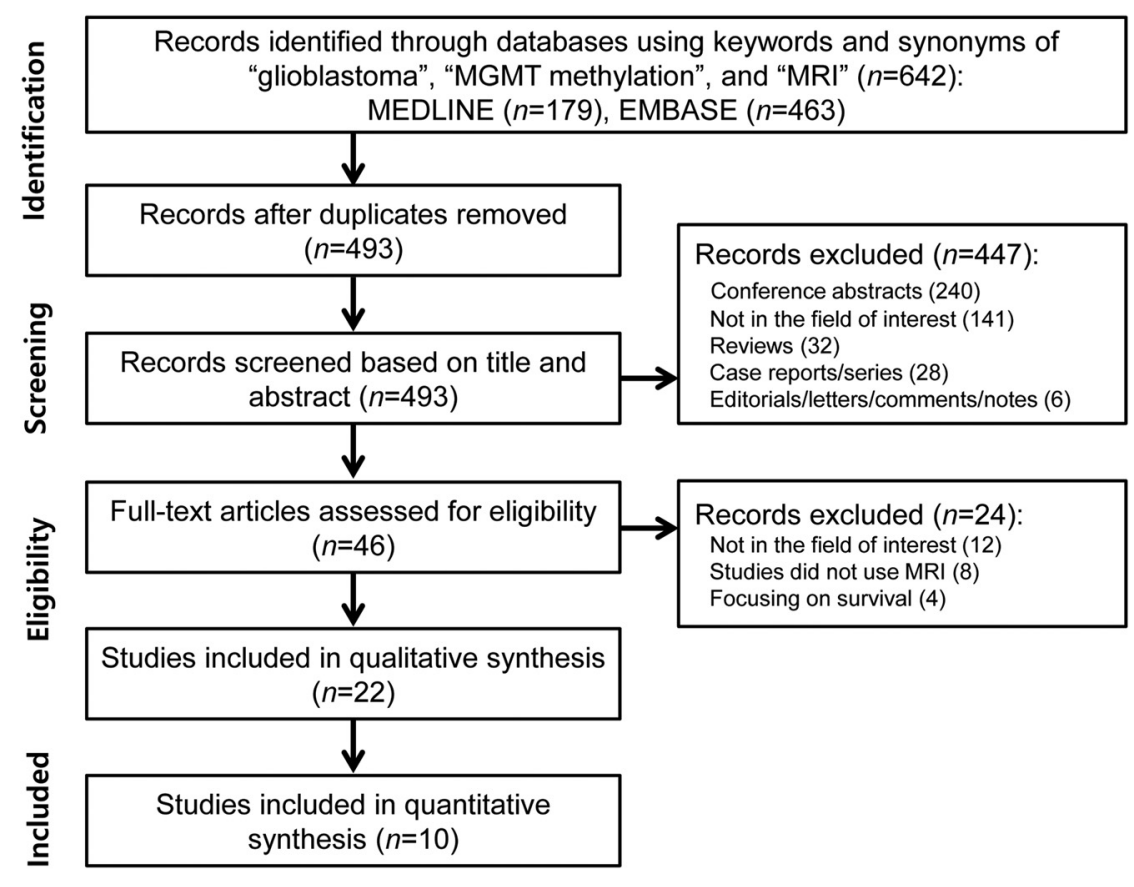

FIG 1. Flow diagram of the study-selection process.

ing for the prediction of MGMT promoter methylation in patients with newly diagnosed glioblastoma were the primary outcomes of the study. To identify their sensitivities and specificities, we reconstructed $2 \times 2$ tables from the included studies. If the diagnostic performances of several MR imaging techniques or multiple readers were assessed separately, the results with higher diagnostic performance were chosen.

Heterogeneity was evaluated by means of the following: 1) the Cochran $\mathrm{Q}$ test, with $P<.05$ indicating the presence of heterogeneity; 2) the Higgins inconsistency index $\left(\mathrm{I}^{2}\right)$ test, with the degree of heterogeneity being interpreted as follows: $\mathrm{I}^{2}=0 \%-40 \%$, heterogeneity might not be important; $30 \%-60 \%$, moderate heterogeneity may be present; $50 \%-90 \%$, substantial heterogeneity may be present; and 75\%-100\%, considerable heterogeneity ${ }^{37} ; 3$ ) visual assessment of a coupled forest plot for the presence of a threshold effect - that is, a positive correlation between sensitivity and the false-positive rate; and 4) a Spearman correlation coefficient of $>0.6$ implying a considerable threshold effect. ${ }^{38}$

The pooled summary estimates of sensitivity and specificity were calculated using hierarchic logistic regression modelingthat is, bivariate and hierarchic summary receiver operating characteristic (HSROC) modeling. ${ }^{39-41}$ An HSROC curve with 95\% confidence and prediction regions was plotted, and the area under the HSROC curve was calculated. Publication bias was analyzed using the Deeks funnel plot, and statistical significance was tested using the Deeks asymmetry test. ${ }^{42}$

A meta-regression was performed to explain the effects of study heterogeneity. The following covariates were analyzed for the bivariate model: 1) the percentage of MGMT promoter methylation in the study population $(<49.5 \%$ [median value of the included studies] versus $\geq 49.5 \%$ ); 2 ) mean age (younger than 59 years [median value of the included studies] versus 59 years or older); 3 ) number of MR imaging readers (1 versus $\geq 2$ ); 4) blinding to the reference standard for MR imaging readers; 5) magnetic field strength (3T versus $1.5 \mathrm{~T}$ ); and 6) the use of radiomics approaches. Subgroup analyses according to studies using apparent diffusion coefficient and perfusion were conducted. In addition, sensitivity analyses were reassessed with the exclusion of 1 study with a lower diagnostic performance.

All statistical meta-analyses were performed by one of the authors (C.H.S., with 4 years of experience in conducting systematic reviews and meta-analyses) using the "midas" and "metandi" modules in STATA 10.0 (StataCorp, College Station, Texas) and the "mada" package in R statistical and computing software, Version 3.4.1 (http:// www.r-project.org). $P$ values $<.05$ indicated statistical significance.

\section{RESULTS}

\section{Literature Search}

The study selection process is described in Fig 1 and the On-line Appendix. Twenty-two original articles evaluating the imaging features of MGMT promoter methylated glioblastoma, including 2199 patients, were included in this study. ${ }^{11-32}$

\section{Characteristics of the Included Studies}

The patient and study characteristics of the included studies are listed in On-line Table 1. The size of the study populations ranged from 17 to 406 patients. Twenty-one of 22 studies included patients with newly diagnosed glioblastoma (WHO grade IV), and 1 study included high-grade glioma (WHO grades III and IV). ${ }^{25}$ One study was prospective in design. ${ }^{26}$ Twenty studies were retrospective, ${ }^{11-25,27,28,30-32}$ and 1 study did not explicitly state the design. ${ }^{29}$ Study enrollment was consecutive in 3 studies $^{16,18,25}$ and not explicit in the remaining studies. Fifteen of 22 studies used methylation-specific polymerase chain reaction as a reference standard to detect MGMT methylation, ${ }^{11,14-16,19-24,27,28,30-32}$ with the other studies using DNA methylation analysis, ${ }^{18}$ pyrosequencing, ${ }^{13}$ immunohistochemical staining, ${ }^{29}$ or multiplex ligation-dependent probe amplification, ${ }^{26}$ with the method not being stated in 3 studies. ${ }^{12,17,25}$

\section{MR Imaging Characteristics of the Included Studies}

The MR imaging characteristics are shown in On-line Table 2. Ten studies used 3T scanners, ${ }^{12,13,15,16,18-21,23,25} 5$ studies used $1.5 \mathrm{~T}$ scanners, ${ }^{22,24,29-31} 3$ studies used $1.5 \mathrm{~T}$ and $3 \mathrm{~T}$ scanners, ${ }^{17,27,28}$ and 4 studies did not state the scanner strength. ${ }^{11,14,26,32}$ A variety of different MR imaging techniques was used to predict MGMT promoter methylation: conventional MR imaging in 11 studies, ${ }^{1,13,14,17,26-32}$ DWI in 4 studies, ${ }^{19,20,22,24} \mathrm{CT} / \mathrm{DWI} / \mathrm{DTI} / \mathrm{DSC}$ in 1 study, ${ }^{25} \mathrm{DWI} / \mathrm{DSC} / \mathrm{SWI}$ in 1 study, ${ }^{18}$ DCE/DTI in 1 study, ${ }^{21}$ DWI/arterial spin-labeling in 1 study, ${ }^{12}$ DSC in 1 study, ${ }^{23}$ DCE in 1 study, ${ }^{16}$ and amide proton transfer in 1 study. ${ }^{15}$ Five studies used radiomics approaches from machine learning-based classification. ${ }^{13,14,17,18,31}$ MR imaging 
was interpreted by 1-3 readers, with the level of experience of the readers ranging from 3 to 25 years. In 14 of 22 studies, the readers were blinded to the MGMT promoter methylation status of patients $^{11-14,16,17,20,21,25,28-32}$; however, this was not stated in the remaining studies.

\section{Quality Assessment}

The results of the quality assessment according to the QUADAS-2 criteria are described in On-line Fig 1 . The quality of the studies was considered moderate, with 14 of the 22 studies satisfying at least 4 of the 7 QUADAS-2 domains. In the patient-selection domain, 20 of 22 studies were considered to have an unclear risk of bias because of nonconsecutive enrollment. ${ }^{11-15,17,19-32}$ In the index test domain, there was an unclear risk of bias in 8 studies because it was unclear whether MR imaging analysis was performed blinded to the reference standard. ${ }^{15,18,19,22-24,26,27}$ In the reference standard domain, the reference standard was not reported in 3 studies. ${ }^{12,17,25}$ In the flow and timing domain, the time intervals between MR imaging and the reference standard were not reported in 15 studies. ${ }^{1-14,16,17,19,20,25,27-32}$

\section{Imaging Features of MGMT Promoter Methylated Glioblastoma: A Systematic Review}

Studies using conventional MR imaging demonstrated a variety of imaging features of MGMT promoter methylated glioblastoma (On-line Table 3). Two such studies revealed that MGMT promoter methylated glioblastoma showed less edema than MGMT promoter unmethylated glioblastoma. ${ }^{27,29}$ One study showed that mixed-nodular enhancement was significantly more frequent in MGMT promoter methylated glioblastoma, ${ }^{32}$ while ring enhancement was more frequent in MGMT promoter unmethylated glioblastoma. ${ }^{30,32}$ One study also showed that MGMT promoter methylated glioblastoma was lateralized to the left hemisphere, while MGMT promoter unmethylated glioblastoma was lateralized to the right hemisphere. ${ }^{27}$ Furthermore, 1 study reported that glioblastoma with a higher rate of MGMT promoter protein expression (>20\%) was less necrotic than glioblastoma negative for, or with a lower rate of, $M G M T$ promoter protein expression. ${ }^{26}$

On DWI, MGMT promoter methylated glioblastoma showed higher ADC values than MGMT promoter unmethylated glioblastoma. ${ }^{12,25}$ In addition, MGMT promoter methylated glioblastoma also had a higher minimum ADC value than MGMT promoter unmethylated glioblastoma. ${ }^{20,24}$ Regarding perfusion, studies using DSC demonstrated that the normalized relative tumor blood volume was significantly lower in MGMT promoter methylated glioblastoma than in MGMT promoter unmethylated glioblastoma. ${ }^{23}$ In addition, studies using arterial spin-labeling demonstrated that relative cerebral blood flow was also significantly lower in MGMT promoter methylated glioblastoma than in MGMT promoter unmethylated glioblastoma. ${ }^{12}$

\section{Diagnostic Performance of MR Imaging for Prediction of MGMT Promoter Methylation: A Meta-Analysis}

Ten original articles, which included 753 patients, evaluated the diagnostic performance of MR imaging in the imaging prediction of MGMT promoter methylation in patients with glioblastoma. ${ }^{12-15,17,18,21,23,24,30}$ The sensitivities and specificities of the individual studies were $56 \%$ to $90 \%$ and $61 \%$ to $91 \%$, respectively. A $Q$ test demonstrated that heterogeneity was absent $(\mathrm{Q}=0.222$, $P=.447)$. The Higgins $\mathrm{I}^{2}$ statistic revealed moderate heterogeneity regarding both the sensitivity $\left(\mathrm{I}^{2}=71.02 \%\right)$ and specificity $\left(\mathrm{I}^{2}=65.71 \%\right)$. A coupled forest plot of the sensitivity and specificity showed no threshold effect (Fig 2). The Spearman correlation coefficient between the sensitivity and false-positive rate was -0.285 (95\% CI, $-0.775-0.420)$, also demonstrating no threshold effect.

The summary sensitivity for all 10 studies was 79\% (95\% CI, $72 \%-85 \%)$, and the summary specificity was $78 \%$ (95\% CI, 71\%$84 \%$; Fig 2). There was a small difference between the $95 \%$ confidence and prediction regions in the HSROC curve, demonstrating a low possibility for the presence of heterogeneity among the studies (On-line Fig 2). The area under the HSROC curve was 0.86 (95\% CI, 0.82-0.88). The Deeks funnel plot showed that the likelihood of publication bias was low $(P=.40$ for the slope coefficient; On-line Fig 3).

\section{Exploration of the Heterogeneity}

A meta-regression was performed to explain the causes of heterogeneity (On-line Table 4). Among the potential covariates, MGMT promoter methylation and mean age were associated with heterogeneity. Number of MR imaging readers, blinding to the reference standard for MR imaging readers, magnetic field strength, and studies using a radiomics approach were not shown to be significant factors affecting the heterogeneity.

Subgroup analysis according to studies using ADC showed that the summary sensitivity was $83 \%(95 \% \mathrm{CI}, 71 \%-96 \%)$ and the summary specificity was $84 \%$ (95\% CI, 74\%-95\%). ${ }^{12,24}$ Subgroup analysis according to studies using perfusion showed that the summary sensitivity was $72 \%(95 \% \mathrm{CI}, 57 \%-88 \%)$ and the summary specificity was $82 \%$ (95\% CI, $72 \%-92 \%) .{ }^{12,21,23}$

One study showed a relatively low diagnostic performance (a sensitivity of $57 \%$ and specificity of $61 \%$ ) in comparison with the other studies. ${ }^{18}$ When a sensitivity analysis was performed with the exclusion of this study, ${ }^{18}$ the heterogeneity in the sensitivity and specificity was resolved, with a recalculated pooled sensitivity of $82 \%$ ( $95 \%$ CI, $77 \%-86 \% ; \mathrm{I}^{2}=28.36 \%$ ) and a specificity of $80 \%$ (95\% CI, 74\%-86\%; $\left.\mathrm{I}^{2}=36.43 \%\right)$.

\section{DISCUSSION}

Our study found that MGMT promoter methylated glioblastoma is likely to show less edema, high ADC, and low perfusion. In addition, this study revealed that the diagnostic performance of MR imaging for prediction of MGMT promoter methylation in patients with newly diagnosed glioblastoma was within a clinically acceptable range, even though various MR imaging techniques were used. The summary sensitivity was $79 \%$ (95\% CI, $72 \%-$ $85 \%)$, the summary specificity was $78 \%$ (95\% CI, $71 \%-84 \%)$, and the area under the HSROC curve was 0.86 (95\% CI, 0.820.88 ). In the meta-regression, heterogeneity was demonstrated in MGMT promoter methylation and mean age. In the sensitivity analysis, the heterogeneities of the sensitivity and specificity were resolved by removal of 1 study with outlying values, resulting in a recalculated pooled sensitivity of $82 \%(95 \% \mathrm{CI}, 77 \%-86 \%)$ and a specificity of $80 \%$ (95\% CI, 74\%-86\%). Despite the variety of MR 


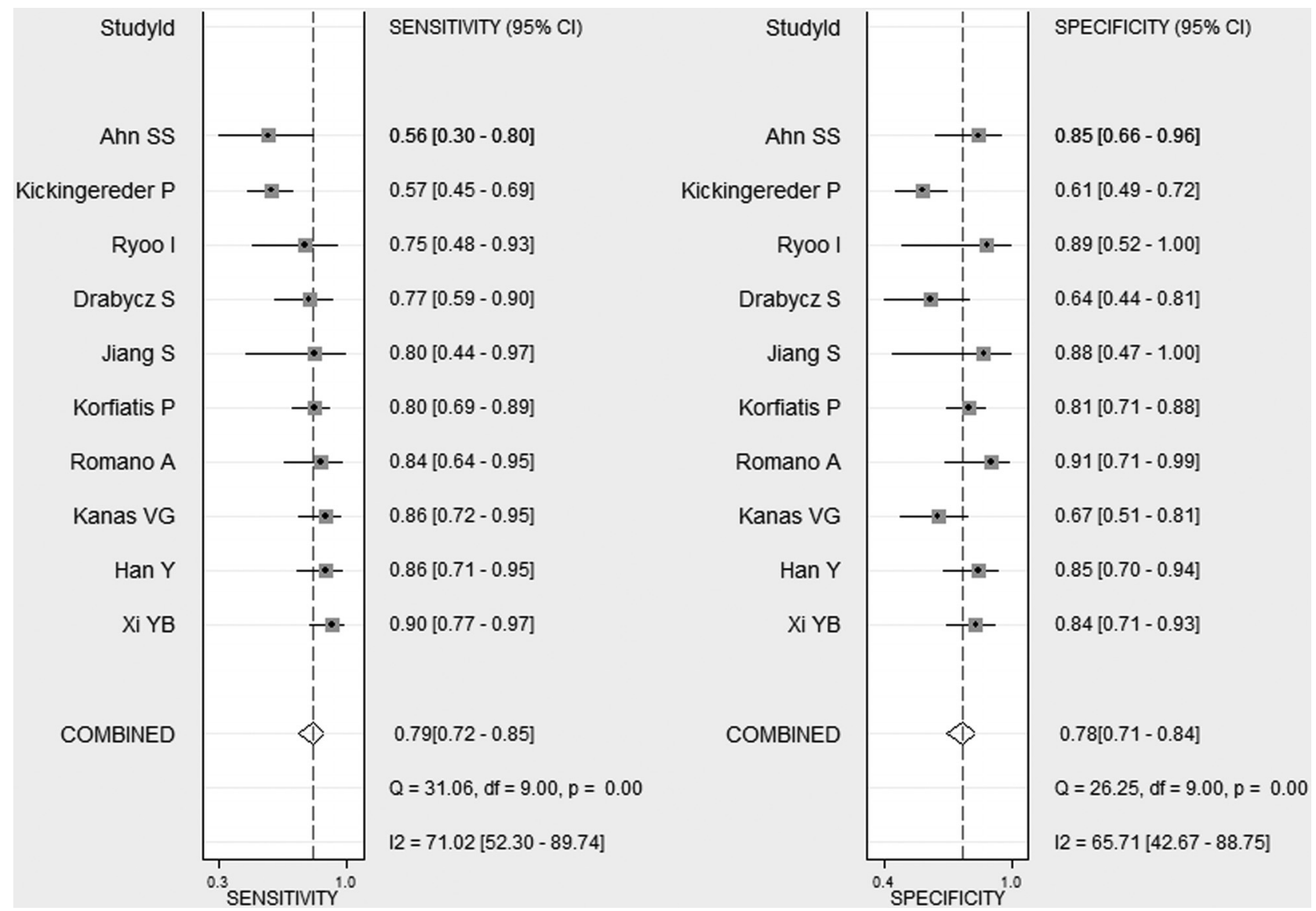

FIG 2. Coupled forest plots of the pooled sensitivity and specificity for the diagnostic performance of MR imaging for prediction of MGMT promoter methylation in patients with newly diagnosed glioblastoma. Numbers are pooled estimates with $95 \%$ confidence intervals in parentheses and horizontal lines indicating $95 \%$ Cls.

imaging techniques used, MR imaging in patients with newly diagnosed glioblastoma was shown to have the potential for noninvasive prediction of MGMT promoter methylation.

Imaging prediction of MGMT promoter methylated glioblastoma has not been widely used because of a lack of standardization in reporting, lack of standardization in imaging sequence, and uncertainty in terms of the criterion standard pathologic diagnosis of MGMT methylation status. In our study, we found that MGMT promoter methylated glioblastoma is likely to show less edema, high ADC, and low perfusion. In addition, studies using $\mathrm{ADC}$ or perfusion showed clinically acceptable diagnostic performance to predict MGMT promoter methylated glioblastoma. Therefore, imaging sequences including ADC or perfusion and corresponding reports of the results should be included. In terms of the reference standard, 15 of 22 studies used methylation-specific polymerase chain reaction. Therefore, we cautiously recommend methylation-specific polymerase chain reaction for the detection of MGMT methylation status. Although a variety of imaging sequences or reference standards was used, we believe our study may help in guiding future research.

Although there are multiple studies describing the distinctive imaging features of MGMT promoter methylated glioblastoma, ${ }^{16,19-24,26,27,29,30,32}$ some discrepancies do exist among them. In our understanding, an exact mechanism for the imaging prediction of MGMT promoter methylation has not been estab- lished. The present study tried to focus on the imaging features of $M G M T$ promoter methylated glioblastoma and the overall diagnostic performance of the various MR imaging techniques. We found that MGMT promoter methylated glioblastoma is likely to show less edema, high ADC, and low perfusion. The potential association between the imaging features and the overall diagnostic performance of MR imaging for the determination of MGMT promoter methylation status may have a predictive value for the evaluation of treatment response in newly diagnosed glioblastoma. This study revealed that MR imaging has a clinically acceptable diagnostic performance in the prediction of MGMT promoter methylation, with a summary sensitivity of $79 \%$ (95\% CI, $72 \%-85 \%)$ and a summary specificity of $78 \%$ (95\% CI, $71 \%-$ $84 \%)$. Therefore, MR imaging in patients with newly diagnosed glioblastoma may potentially provide a noninvasive comprehensive and repetitive assessment for MGMT promoter methylation. However, due to the paucity of information regarding imaging features of MGMT promoter methylated glioblastoma, further study will be needed.

MGMT promoter methylation has been proposed as a predictive biomarker for benefit from alkylating agent chemotherapy in patients with isocitrate dehydrogenase (IDH) wild-type, but not $I D H$-mutant gliomas. This is due to the close correlation between IDH mutations and the presence of MGMT promoter methylation. ${ }^{43,44}$ One of the explanations for this correlation is that $I D H$ 
mutation is associated with a CpG island methylator phenotype in glioma. ${ }^{43,44}$ Therefore, the determination of MGMT promoter methylation status is clinically relevant in $I D H$ wild-type lower grade gliomas as well as $I D H$ wild-type glioblastoma. However, only $20 \%-30 \%$ of lower grade gliomas are $I D H$ wild-type; therefore, due to the paucity of information reported so far, we could not include $I D H$ wild-type lower grade gliomas. Further studies will be needed to answer the clinically relevant question.

The current study highlights 4 studies that evaluated the diagnostic performance of radiomics approaches in combination with machine-learning methods. ${ }^{13,14,17,18}$ With increases in the use of combined multiple imaging parameters to predict molecular characteristics, many researchers have used advanced statistical methods to increase the performance of such multiparametric imaging parameters. In particular, machine learning-based classification models have been popular; these aim to establish predictive models for molecular parameters by integrating all the available information from various MR imaging features. ${ }^{18}$ The present study revealed a summary sensitivity of 79\% (95\% CI, $70 \%-88 \%)$ and a summary specificity of $73 \%$ (95\% CI, 66\%$81 \%$ ) for radiomics approaches, which is within a clinically acceptable range. However, studies using radiomics approaches showed slightly lower summary specificity than the other studies without statistical significance (87\% [95\% CI, 80\%-94\%]; $P=$ .42 ). A possible reason for this significant difference is that radiomics approaches, which are data-driven, may be extensively affected by data quality. In addition, the step-by-step processes of radiomics approaches, which include imaging technique, feature extraction, feature selection, and classification by machine learning, vary substantially across institutions. Moreover, 3 studies used conventional MR imaging, ${ }^{13,14,17}$ while another study used multiparametric and multiregional MR imaging, including advanced techniques. ${ }^{18}$ Further effort to achieve the standardization of radiomics approaches is required.

This study revealed moderate heterogeneity across the included studies in both the sensitivity $\left(\mathrm{I}^{2}=71.02 \%\right)$ and the specificity $\left(\mathrm{I}^{2}=65.71 \%\right)$ measures. A meta-regression showed that MGMT promoter methylation and mean age were associated with heterogeneity. In addition, sensitivity analysis also demonstrated that the heterogeneities in the sensitivity and specificity were resolved when a study with outlying values was removed $\left(\mathrm{I}^{2}=\right.$ $28.36 \%$ and $\mathrm{I}^{2}=36.43 \%$, respectively). Although our meta-regression and sensitivity analysis may have explained some of the heterogeneity, further heterogeneity remains unexplained. A wide variety of MR imaging techniques was used to predict MGMT promoter methylation across the included studies: conventional MR imaging, ${ }^{13,14,17,30}$ DWI/DSC/SWI, ${ }^{18}$ DWI/arterial spin-labeling, ${ }^{12}$ DCE/DTI, ${ }^{21}$ DWI,${ }^{24}$ DSC, ${ }^{23}$ and amide proton transfer. ${ }^{15}$ The use of these different MR imaging techniques across the included studies is considered a major cause of study heterogeneity. To use MR imaging as a potential imaging predictor for molecular classification, the standardization of MR imaging acquisition, processing, and image analysis should be established.

This study is subject to several limitations. First, 22 studies were included in the systematic review; however, only 10 studies were included in the meta-analysis. In terms of imaging features of MGMT promoter methylated glioblastoma, a small number of studies for each technique were included. However, these studies were the only currently available articles, and future studies will be needed. Second, the included studies used a variety of different MR imaging techniques to predict MGMT promoter methylation, and heterogeneity was reported in the sensitivity and specificity. To identify the source of the heterogeneity, we conducted a meta-regression and sensitivity analysis. We conducted our systematic review and meta-analysis using robust methodology (hierarchic logistic regression modeling ${ }^{39-41}$ ) and reported the study results according to prestigious guidelines: PRISMA, ${ }^{33}$ the Agency for Healthcare Research and Quality, ${ }^{45}$ and the Handbook for Systematic Reviews of Diagnostic Test Accuracy published by the Cochrane Collaboration. ${ }^{46}$

\section{CONCLUSIONS}

MGMT promotor methylated glioblastoma is likely to show less aggressive imaging features than MGMT promotor unmethylated glioblastoma. Despite the variety of different MR imaging techniques used, MR imaging in patients with newly diagnosed glioblastoma was shown to have the potential to predict MGMT promoter methylation noninvasively.

\section{REFERENCES}

1. Esteller M, Herman JG. Generating mutations but providing chemosensitivity: the role of O6-methylguanine DNA methyltransferase in human cancer. Oncogene 2004;23:1-8 CrossRef Medline

2. Hegi ME, Diserens AC, Gorlia T, et al. MGMT gene silencing and benefit from temozolomide in glioblastoma. N Engl J Med 2005;352: 997-1003 CrossRef Medline

3. Esteller M, Garcia-Foncillas J, Andion E, et al. Inactivation of the DNA-repair gene MGMT and the clinical response of gliomas to alkylating agents. N Engl J Med 2000;343:1350-54 CrossRef Medline

4. Brandes AA, Tosoni A, Franceschi E, et al. Recurrence pattern after temozolomide concomitant with and adjuvant to radiotherapy in newly diagnosed patients with glioblastoma: correlation with MGMT promoter methylation status. J Clin Oncol 2009;27:1275-79 CrossRef Medline

5. Weller M, Tabatabai G, Kastner B, et al; DIRECTOR Study Group. MGMT promoter methylation is a strong prognostic biomarker for benefit from Dose-Intensified Temozolomide Rechallenge in Progressive Glioblastoma: the DIRECTOR trial. Clin Cancer Res 2015; 21:2057-64 CrossRef Medline

6. Bell EH, Pugh SL, McElroy JP, et al. Molecular-based recursive partitioning analysis model for glioblastoma in the temozolomide era: a correlative analysis based on NRG oncology RTOG 0525. JAMA Oncol 2017;3:784-92 CrossRef Medline

7. Perry JR, Laperriere N, O'Callaghan CJ, et al; Trial Investigators. Short-course radiation plus temozolomide in elderly patients with glioblastoma. N Engl J Med 2017;376:1027-37 CrossRef Medline

8. Zarnett OJ, Sahgal A, Gosio J, et al. Treatment of elderly patients with glioblastoma: a systematic evidence-based analysis. JAMA Neurol 2015;72:589-96 CrossRef Medline

9. Brandes AA, Franceschi E, Tosoni A, et al. MGMT promoter methylation status can predict the incidence and outcome of pseudoprogression after concomitant radiochemotherapy in newly diagnosed glioblastoma patients. J Clin Oncol 2008;26:2192-97 CrossRef Medline

10. Weller M, Cloughesy T, Perry JR, et al. Standards of care for treatment of recurrent glioblastoma: are we there yet? Neuro Oncol 2013; 15:4-27 CrossRef Medline

11. Li HY, Sun CR, He M, et al. Correlation between tumor location and clinical properties of glioblastomas in frontal and temporal lobes. World Neurosurg 2018;112:e407-14 CrossRef Medline

12. Han Y, Yan LF, Wang XB, et al. Structural and advanced imaging in 
predicting MGMT promoter methylation of primary glioblastoma: a region of interest based analysis. BMC Cancer 2018;18:215 CrossRef Medline

13. Xi YB, Guo F, Xu ZL, et al. Radiomics signature: a potential biomarker for the prediction of MGMT promoter methylation in glioblastoma. J Magn Reson Imaging 2018;47:1380 - 87 CrossRef Medline

14. Kanas VG, Zacharaki EI, Thomas GA, et al. Learning MRI-based classification models for MGMT methylation status prediction in glioblastoma. Comput Methods Programs Biomed 2017;140:249-57 CrossRef Medline

15. Jiang S, Rui Q, Wang Y, et al. Discriminating MGMT promoter methylation status in patients with glioblastoma employing amide proton transfer-weighted MRI metrics. Eur Radiol 2018;28:2115-23 CrossRef Medline

16. Choi YS, Ahn SS, Lee HJ, et al. The initial area under the curve derived from dynamic contrast-enhanced MRI improves prognosis prediction in glioblastoma with unmethylated MGMT promoter. AJNR Am J Neuroradiol 2017;38:1528-35 CrossRef Medline

17. Korfiatis P, Kline TL, Coufalova L, et al. MRI texture features as biomarkers to predict MGMT methylation status in glioblastomas. Med Phys 2016;43:2835-44 CrossRef Medline

18. Kickingereder P, Bonekamp D, Nowosielski M, et al. Radiogenomics of glioblastoma: machine learning-based classification of molecular characteristics by using multiparametric and multiregional MR imaging features. Radiology 2016;281:907-18 CrossRef Medline

19. Choi YS, Ahn SS, Kim DW, et al. Incremental prognostic value of ADC histogram analysis over MGMT promoter methylation status in patients with glioblastoma. Radiology 2016;281:175-84 CrossRef Medline

20. Rundle-Thiele D, Day B, Stringer B, et al. Using the apparent diffusion coefficient to identifying MGMT promoter methylation status early in glioblastoma: importance of analytical method. J Med Radiat Sci 2015;62:92-98 CrossRef Medline

21. Ahn SS, Shin NY, Chang JH, et al. Prediction of methylguanine methyltransferase promoter methylation in glioblastoma using $\mathrm{dy}$ namic contrast-enhanced magnetic resonance and diffusion tensor imaging. J Neurosurg 2014;121:367-73 CrossRef Medline

22. Sunwoo L, Choi SH, Park CK, et al. Correlation of apparent diffusion coefficient values measured by diffusion MRI and MGMT promoter methylation semiquantitatively analyzed with MS-MLPA in patients with glioblastoma multiforme. J Magn Reson Imaging 2013; 37:351-58 CrossRef Medline

23. Ryoo I, Choi SH, Kim JH, et al. Cerebral blood volume calculated by dynamic susceptibility contrast-enhanced perfusion MR imaging: preliminary correlation study with glioblastoma genetic profiles. PLoS One 2013;8:e71704 CrossRef Medline

24. Romano A, Calabria LF, Tavanti F, et al. Apparent diffusion coefficient obtained by magnetic resonance imaging as a prognostic marker in glioblastomas: correlation with MGMT promoter methylation status. Eur Radiol 2013;23:513-20 CrossRef Medline

25. Moon WJ, Choi JW, Roh HG, et al. Imaging parameters of high grade gliomas in relation to the MGMT promoter methylation status: the CT, diffusion tensor imaging, and perfusion MR imaging. Neuroradiology 2012;54:555-63 CrossRef Medline

26. Iliadis G, Kotoula V, Chatzisotiriou A, et al. Volumetric and MGMT parameters in glioblastoma patients: survival analysis. BMC Cancer 2012;12:3 CrossRef Medline

27. Ellingson BM, Cloughesy TF, Pope WB, et al. Anatomic localization of O6-methylguanine DNA methyltransferase (MGMT) promoter methylated and unmethylated tumors: a radiographic study in 358 de novo human glioblastomas. Neuroimage 2012;59: 908-16 CrossRef Medline

28. Carrillo JA, Lai A, Nghiemphu PL, et al. Relationship between tumor enhancement, edema, IDH1 mutational status, MGMT promoter methylation, and survival in glioblastoma. AJNR Am J Neuroradiol 2012;33:1349-55 CrossRef Medline
29. Li WB, Tang K, Zhang W, et al. Relationship between magnetic resonance imaging and molecular pathology in patients with glioblastoma multiforme. Chin Med J (Engl) 2011;124:2589-92 Medline

30. Drabycz S, Roldán G, de Robles P, et al. An analysis of image texture, tumor location, and MGMT promoter methylation in glioblastoma using magnetic resonance imaging. Neuroimage 2010;49:1398-405 CrossRef Medline

31. Levner I, Drabycz S, Roldan G, et al. Predicting MGMT methylation status of glioblastomas from MRI texture. Med Image Comput Comput Assist Interv 2009;12(Pt 2):522-30 Medline

32. Eoli M, Menghi F, Bruzzone MG, et al. Methylation of O6-methylguanine DNA methyltransferase and loss of heterozygosity on 19q and/or $17 \mathrm{p}$ are overlapping features of secondary glioblastomas with prolonged survival. Clin Cancer Res 2007;13:2606-13 CrossRef Medline

33. Liberati A, Altman DG, Tetzlaff J, et al. The PRISMA statement for reporting systematic reviews and meta-analyses of studies that evaluate health care interventions: explanation and elaboration. Ann Intern Med 2009;151:W65-94 Medline

34. Louis DN, Ohgaki H, Wiestler OD, et al. The 2007 WHO classification of tumours of the central nervous system. Acta Neuropathol 2007;114:97-109 CrossRef Medline

35. Louis DN, Perry A, Reifenberger G, et al. The 2016 World Health Organization Classification of Tumors of the Central Nervous System: a summary. Acta Neuropathol 2016;131:803-20 CrossRef Medline

36. Whiting PF, Rutjes AW, Westwood ME, et al; QUADAS-2 Group. QUADAS-2: a revised tool for the quality assessment of diagnostic accuracy studies. Ann Intern Med 2011;155:529-36 CrossRef Medline

37. Higgins J, Green S. Cochrane Handbook for Systematic Reviews of Interventions, Version 5.1.0. The Cochrane Collaboration. http:// handbook-5-1.cochrane.org/. Updated March 2011. Accessed October 9,2017

38. Devillé WL, Buntinx F, Bouter LM, et al. Conducting systematic reviews of diagnostic studies: didactic guidelines. BMC Med Res Methodol 2002;2:9 CrossRef Medline

39. Suh $\mathrm{CH}$, Park SH. Successful publication of systematic review and meta-analysis of studies evaluating diagnostic test accuracy. Korean J Radiol 2016;17:5-6 CrossRef Medline

40. Kim KW, Lee J, Choi SH, et al. Systematic review and meta-analysis of studies evaluating diagnostic test accuracy: a practical review for clinical researchers, Part 1: general guidance and tips. Korean J Radiol 2015;16:1175-87 CrossRef Medline

41. Lee J, Kim KW, Choi SH, et al. Systematic review and meta-analysis of studies evaluating diagnostic test accuracy: a practical review for clinical researchers, Part 2: statistical methods of meta-analysis. Korean J Radiol 2015;16:1188-96 CrossRef Medline

42. Deeks JJ, Macaskill P, Irwig L. The performance of tests of publication bias and other sample size effects in systematic reviews of diagnostic test accuracy was assessed. J Clin Epidemiol 2005;58:882-93 CrossRef Medline

43. Wick W, Meisner C, Hentschel B, et al. Prognostic or predictive value of MGMT promoter methylation in gliomas depends on IDH1 mutation. Neurology 2013;81:1515-22 CrossRef Medline

44. van den Bent MJ, Erdem-Eraslan L, Idbaih A, et al. MGMT-STP27 methylation status as predictive marker for response to PCV in anaplastic oligodendrogliomas and oligoastrocytomas: a report from EORTC study 26951. Clin Cancer Res 2013;19:5513-22 CrossRef Medline

45. Trikalinos TA, Balion CM, Coleman CI, et al. Chapter 8: meta-analysis of test performance when there is a "gold standard". J Gen Intern Med 2012;27(Suppl 1):S56-66 CrossRef Medline

46. Deeks JJ, Bossuyt PM, Gatsonis C, eds. 2013 Cochrane Handbook for Systematic Reviews of Diagnostic Test Accuracy, Version 1.0.0. The Cochrane Collaboration. http://srdta.cochrane.org/handbook-dtareviews. Accessed October 9, 2017 